\title{
Unintended Pregnancy and Associated Factors Among Women Attending Antenatal Care in Public Hospitals During COVID-19 Pandemic, Southwest Ethiopia: A Cross-Sectional Study
}

\author{
Shegaw Geze Tenaw (D)', Fantaye Chemir (D)', Bitew Tefera Zewudie $\mathbb{D}^{2}$, Bogale Chekole $\mathbb{D}^{2}$, \\ Muche Argaw', Yibelital Mesfin (D)', Mebratu Demissie', Keyredin Nuriye Metebo (D)', Yirgalem Yosef', \\ Daniel Tsega', Haimanot Abebe ${ }^{2}$, Shegaw Tesfa $\mathbb{D}^{2}$, Seblework Abeje ${ }^{3}$ \\ 'Department of Midwifery, College of Medicine and Health Sciences, Wolkite University, Wolkite, Ethiopia; ${ }^{2}$ Department of Nursing, College of \\ Medicine and Health Sciences, Wolkite University, Wolkite, Ethiopia; ${ }^{3}$ Department of Biomedical, College of Medicine and Health Sciences, Wolkite \\ University, Wolkite, Ethiopia
}

Correspondence: Shegaw Geze Tenaw, Department of Midwifery, College of Medicine and Health Sciences, Wolkite University, Wolkite, Ethiopia, Email shegaw.geze@gmail.com

Background: COVID-19 pandemic directly or indirectly increases the burden of unintended pregnancy by limiting women's access to family planning and other reproductive health services. COVID-19 results in extra 15 million unintended pregnancies over a year. Almost all previous studies conducted about unintended pregnancy were before COVID-19 pandemic in Ethiopia. Therefore, the purpose of this study was to assess the prevalence and associated factors of unintended pregnancy during the COVID-19 pandemic among women attending antenatal care in public hospitals of southwest Ethiopia.

Methods: This study was cross-sectional and conducted among women attending antenatal care at public hospitals of southwest Ethiopia from June 14 to July 14, 2021. Data were collected using a face-to-face interview. Factors associated with unintended pregnancy were analyzed using binary and multiple logistic regressions with an adjusted odds ratio and $95 \%$ confidence interval. Finally, the p-value was used as a graded measure of evidence to quantify the degree of significance.

Results: A total of 405 women participated in this study. The overall prevalence of unintended pregnancy was 19.5\% (95\% CI: 1.44-6.92) among women attending antenatal care during COVID-19 pandemic. Of which, 50.6\% were mistimed and 49.4\% unwanted. Urban residence (AOR: 3.1 95\% CI: 1.44-6.92) and not being primary decision-maker (AOR: 2.85 95CI: 1.18-6.88) had high significance with unintended pregnancy. Not having ANC in a previous pregnancy (AOR: 3.40; 95\% CI: $1.02-11.94$ ) and not being exposed to community education about maternal health care (AOR: 2.36; 95\% CI: 1.06-5.27) had medium significance with unintended pregnancy.

Conclusion: One-fifth of women attending antenatal care had unintended pregnancies during the COVID-19 pandemic. Efforts to scale up women's decision-making power on family planning services and access to community education are needed to prevent unintended pregnancy.

Keywords: unintended pregnancy, COVID-19, pandemic, antenatal care, Ethiopia

\section{Background}

Unintended pregnancy refers to a pregnancy that is either mistimed or unwanted. ${ }^{1}$ Unintended pregnancy has been a troubling public health and reproductive health issue, which imposes appreciable adverse consequences on the mother, child, and the public in general. ${ }^{2}$ In addition to abortion and its complications, ${ }^{3,4}$ unintended pregnancy costs to health and social services impose significant emotional distress on women and their families. ${ }^{5}$ Therefore, it is vital to prevent unintended pregnancy based on evidence to save the costs of abortion and its complications and reduce the morbidity and mortality of women and their children. 
Globally 121 million unplanned pregnancies occurred from 2015 to 2019. ${ }^{3}$ A significant proportion (61\%) of these pregnancies ended in abortions each year. Although there has been a decline in unintended pregnancy around the globe, it has been unequal between high-income countries, compared with middle and low-income countries, where 66 unintended pregnancies per 1000 women and 93 per 100 women, respectively. ${ }^{3}$ Despite this general decline and availability of widespread family planning services, the burden of an unintended pregnancy remains high in sub-Saharan Africa. ${ }^{3,6}$ In Ethiopia, the overall prevalence of unintended pregnancy was $28 \%$ before the COVID- 19 pandemic ${ }^{7}$ and during the Covid-19 pandemic in northwest Ethiopia was $47 \%{ }^{8}$

COVID-19 pandemic directly or indirectly increases the burden of unintended pregnancy by limiting women's access to contraceptives and other sexual and reproductive health services in several ways. During this pandemic, women are not visiting health institutions due to lockdown and fears of exposure. The health care providers shift from providing family planning service towards Covid 19 response. ${ }^{9-11}$ One in three women delayed or canceled visiting health care providers for sexual and reproductive health services or had trouble getting their birth control. ${ }^{12}$ It was estimated that a ten percent decline in access to long and short-acting modern contraceptives in middle- and low-income countries. Due to COVID-19, additional 48 million unmet needs for modern contraceptives and 15 million unintended pregnancies occurred over a year. ${ }^{13}$ A study in 37 countries during COVID reported that 1.9 million women and girls lost access to contraceptives during the first half of the years. ${ }^{14}$ According to UNFPA estimation, within one year into the pandemic of Covid-19, 12 million women have seen contraceptive interruptions, which lead to 1.4 million unintended pregnancies. ${ }^{15}$

Although there were several studies about the prevalence and associated factors of unintended pregnancy in Ethiopia, almost all studies were before Covid-19. ${ }^{16-22}$ Given the limited access to family planning services and the preventive strategies of Covid-19 increase the burden of unintended pregnancy, ${ }^{9-11}$ it is vital to study unintended pregnancy to provide evidence-based intervention. Although a recent study estimated unintended pregnancy during Covid-19, it was in a single area, which is not representative of the populations in the country. ${ }^{8}$ Thus, this study aimed to assess unintended pregnancy during the COVID-19 pandemic in public hospitals of southwest Ethiopia.

\section{Methods}

\section{Study Design and Settings}

This was a hospital-based cross-sectional study conducted from June 14 to July 14, 2021, at Worabe Comprehensive Specialized Hospital and Wolkite Specialized Teaching Hospital southwest Ethiopia. Both hospitals render many services for the community, such as in-patient department, outpatient department, antenatal care, labor and delivery, and postnatal care. The hospitals also provide immunization services for infants and family planning services such as long-term and short-term contraceptives and education on family planning services. The annual report of women who attended ANC was 21,960 in both hospitals.

\section{Participants}

The study participants were all pregnant women who attended antenatal care in both hospitals during the study period. We excluded women who were critically ill during the data collection period.

\section{Sample Size and Sampling Technique}

The sample size was determined using a single population proportion formula using the following assumptions: a confidence interval of $95 \%$, the margin of error of $5 \%$, and prevalence of unintended pregnancy $46 \%{ }^{8}$ By adding a $10 \%$ non-response rate, the final sample size became 421 . The study participants were selected using a systematic random sampling technique. We calculated the sampling interval $(K)$ by $K=N / n$, where $N=$ the total average number of pregnant women expected to attend antenatal care per month in both hospitals (1830), $\mathrm{n}=$ the final sample size (421). Accordingly, every 4th woman enrolled for the study based on the sequence of their exit after a check-up. The first study subject was selected using a lottery method among the first four pregnant women attending antenatal care. 


\section{Data Collection Tool and Procedure}

Data were collected by face-to-face interview using a pre-tested structured questionnaire. The questionnaire consisted of socio-demographic characteristics, obstetrics characteristics, and reproductive history-related questions. To assure the quality of data: trained research assistants were collecting the data under the supervision of the principal investigator. Filled questionnaires were checked daily for consistencies and completeness. The reliability of the questionnaire was checked by using Cronbach's alpha value, which was 0.75 .

\section{Data Measurement and Study Variables}

The dependent variable was unintended pregnancy. Independent variables were as follows: socio-demographic characteristics (religion, occupational status, marital status, educational level), obstetrics and reproductive health characteristics (parity, history of abortion, history of stillbirth, and ANC in a previous pregnancy, information on family planning, ever use of contraceptives, spousal communication, primary decision-maker about family planning use), and Community education about maternal health care.

Unintended pregnancy refers to a woman whose pregnancy is either unwanted or mistimed during the time of conception. $^{23}$

Mistimed pregnancy refers to a pregnancy that occurs in women who conceived sooner or later than the desired time. $^{23}$

Unwanted pregnancy refers to a woman who conceived when no children or no more children are desired. ${ }^{23}$

\section{Data Management and Analysis}

Data were cleaned and entered into Epi Data 3.1 and exported to SPSS 25 for analysis. The data was cleaned by running frequency, checking missing values, and checking the presence of outliers. We summarized participant characteristics using proportions of categorical variables and mean with standard deviation (SD) or median with interquartile range (IQR) based on the distribution of data for continuous variables. Normality was checked using the Shapiro-Wilk test. Normality was checked using the Shapiro-Wilk test. Factors associated with the outcome variable were analyzed using binary logistic regression. Bivariate analysis, crude odds ratio with $95 \% \mathrm{CI}$, was used to see the association between each independent variable with the outcome variable. Variables with $p$-value of $\leq 0.25$ in the bivariate analysis were selected for the multivariable logistic regression model. Multi-collinearity was checked to see the linear correlation among the independent variables. Multivariable logistic regression analysis was computed, to control the possible effects of confounders. Model goodness of fitness was tested by Hosmer-Lemeshow statistic. The strength of the association between dependent and independent variables was assessed using an adjusted odds ratio with a $95 \%$ confidence interval. A $p$-value was used to report the level of significance of each independent variable with a dependent variable.

\section{Results}

\section{Socio-Demographic Characteristics}

A total of 405 women participated in the study result a response rate of $96.2 \%$. The mean age \pm SD of the respondents was $27.57 \pm 6.06$. A great majority $(96.8 \%)$ of women were Muslim, $392(96.8 \%)$ were married, more than half 221 $(54.6 \%)$ of the study participants were from urban residence, and $152(37.5 \%)$ had no formal education (Table 1).

\section{Prevalence of Unintended Pregnancy}

A total of 79 (19.5\%) pregnant women reported that their pregnancy was unintended during the COVID-19 pandemic. Of which, 39 (49.4\%) pregnancies were unwanted, and 40 (50.6\%) were mistimed.

\section{Obstetric and Reproductive Health-Related Characteristics}

Of 405 respondents, 91 (22.5\%) were nulliparous, 18 (4.4\%) had a history of abortion, and $12(3 \%)$ had a stillbirth. More than two-third $314(77.5 \%)$ of women had previous ANC follow, and 55 (13.5\%) women have previous pregnancy-related 
Table I Socio-Demographic Characteristics of the Study Participants in Public Hospitals of Southwest Ethiopia, 202I $(n=405)$

\begin{tabular}{|c|c|c|c|}
\hline Variables & Category & Frequency & Percentage (\%) \\
\hline \multirow[t]{3}{*}{ Age } & $15-19$ & 24 & 5.9 \\
\hline & $20-34$ & 293 & 72.3 \\
\hline & $35-49$ & 88 & 21.7 \\
\hline \multirow[t]{3}{*}{ Religion } & Muslim & 388 & 95.8 \\
\hline & Orthodox & 13 & 3.2 \\
\hline & Protestant & 4 & 1.0 \\
\hline \multirow[t]{2}{*}{ Residence } & Urban & 221 & 54.6 \\
\hline & Rural & 184 & 45.4 \\
\hline \multirow[t]{2}{*}{ Marital status } & Married & 392 & 96.8 \\
\hline & Single & 13 & 3.2 \\
\hline \multirow[t]{3}{*}{ Educational status of women } & No formal education & 152 & 37.5 \\
\hline & Primary school & 144 & 35.6 \\
\hline & $\begin{array}{l}\text { Secondary school and } \\
\text { above }\end{array}$ & 109 & 26.9 \\
\hline \multirow[t]{5}{*}{ Occupational status of women } & Housewife & 286 & 70.6 \\
\hline & Government employee & 42 & 10.4 \\
\hline & Merchant & 47 & 11.6 \\
\hline & Daily labor & 17 & 4.2 \\
\hline & Other* & 13 & 3.2 \\
\hline
\end{tabular}

Note: Other*, student and private worker.

complications. Of 405 women, 353 (87.2\%) have information about contraceptives, 218 (53.8\%) used contraceptives before COVID 19 pandemic, and 141 (34.8\%) used contraceptives in the pandemic (Table 2).

\section{Factors Associated with Unintended Pregnancy}

In adjusted analysis, urban residence (AOR: $3.195 \% \mathrm{CI}$ : 1.44-6.92) and not being primary decision-maker (AOR: 2.85 95CI: 1.18-6.88) had high significance with unintended pregnancy. Not having ANC in a previous pregnancy (AOR: 3.40; 95\% CI: 1.02-11.94) and not being exposed to community education about maternal health care (AOR: 2.36 ; $95 \%$ CI: 1.06-5.27) had medium significance with unintended pregnancy. Communicating with a partner about family planning had a low significance with unintended pregnancy $(\mathrm{p}=0.064)$. Having information about contraception $(\mathrm{p}=$ $0.730)$, age below 20 years $(p=0.313)$, ever use contraceptive $(p=0.196)$ had very low significance with unintended pregnancy (Table 3).

\section{Discussion}

This study assessed the prevalence of unintended pregnancy and its associated factors during the COVID 19 pandemic in public hospitals of southwest Ethiopia. We found the prevalence of unintended pregnancy $19.5 \%$ (95\% CI: $15.8 \%, 23.4 \%)$.

The prevalence of unintended pregnancy in this study is lower than the study finding at Gondar $47.17 \%),{ }^{8}$ Maichew $(29.7 \%),{ }^{22}$ and southern Ethiopia (36.6\%). ${ }^{24}$ This finding was also lower than the study in Ghana $(40 \%),{ }^{25}$ and Nigeria 
Table 2 Obstetrics and Reproductive Health-Related Characteristics of Women Attending ANC in Public Hospitals of Southwest Ethiopia, $2021(n=405)$

\begin{tabular}{|c|c|c|c|}
\hline Variables & Category & Frequency & Percentage (\%) \\
\hline \multirow[t]{2}{*}{ Gravidity } & Primigravida & 91 & 22.5 \\
\hline & Multigravida & 314 & 77.5 \\
\hline \multirow[t]{3}{*}{ Parity } & Nulliparous & 91 & 22.5 \\
\hline & Primiparous & 74 & 18.2 \\
\hline & Multiparous & 240 & 59.2 \\
\hline \multirow[t]{2}{*}{ History of abortion } & Yes & 18 & 4.4 \\
\hline & No & 387 & 95.6 \\
\hline \multirow[t]{2}{*}{ History of still birth } & Yes & 12 & 3.0 \\
\hline & No & 393 & 97 \\
\hline \multirow[t]{2}{*}{ Previous history of ANC } & Yes & 314 & 77.5 \\
\hline & No & 91 & 22.5 \\
\hline \multirow[t]{2}{*}{ Information about contraception } & Yes & 353 & 87.2 \\
\hline & No & 52 & 12.8 \\
\hline \multirow{2}{*}{$\begin{array}{l}\text { Community education about maternity health } \\
\text { services }\end{array}$} & Yes & 218 & 53.8 \\
\hline & No & 187 & 46.2 \\
\hline \multirow[t]{2}{*}{ Primary decision on family planning use } & Women & 309 & 76.3 \\
\hline & Her husband & 96 & 23.7 \\
\hline \multirow[t]{2}{*}{ Spousal communication about family planning } & Yes & 240 & 59.3 \\
\hline & No & 165 & 40.7 \\
\hline \multirow[t]{2}{*}{ Ever use Contraceptive } & Yes & 211 & 52.1 \\
\hline & No & 194 & 47.8 \\
\hline
\end{tabular}

$(35.9 \%) .{ }^{26}$ The variation might also be due to unstable pregnancy intention: once the pregnancy happens, there is a tendency to be reported as intended. ${ }^{27}$ However, this finding is comparable with a study in South Asian countries $(19.1 \%)^{28}$ and Arba Minch town in Ethiopia (19.4\%). ${ }^{29}$

Women who resided in urban areas were three times more likely to have an unintended pregnancy than their counterparts. However, the urban residence was a protective factor for unintended pregnancy in a study done at Tepi General Hospital in Ethiopia. ${ }^{30}$ This inconsistency might be due to time variation. Nowadays, due to the influence of globalization, urban women are more often engaged in sexual activity and start it before marriage. Therefore, if pregnancy occurs, it is more likely to be unintended. In this study, $84.6 \%$ of single women had unintended pregnancies, whereas only $17.3 \%$ of married women had unintended pregnancies.

Consistent with a study at Gondar in Ethiopia during the COVID-19 pandemic, women who were not the primary decision maker for family planning use were more likely that the pregnancy was unintended. ${ }^{8}$ This finding indicates the importance of having the autonomy to decide on family planning services to get the contraceptive methods at the right time.

Women who had no ANC in previous pregnancy were 3.4 times more likely to experience unintended pregnancy than their counterparts. Antenatal care provides the opportunity to get information about family planning services and when to plan another pregnancy. 
Table 3 Factors Associated with Unintended Pregnancy in Women Attending ANC in Public Hospitals of Southwest Ethiopia, 202I $(n=405)$

\begin{tabular}{|c|c|c|c|c|c|c|}
\hline \multirow[t]{2}{*}{ Variable } & \multirow[t]{2}{*}{ Category } & \multicolumn{2}{|c|}{$\begin{array}{l}\text { Unintended } \\
\text { Pregnancy }\end{array}$} & \multicolumn{2}{|c|}{ 95\% Confidence Interval } & \multirow[t]{2}{*}{ p-value } \\
\hline & & Yes (\%) & No (\%) & COR & AOR & \\
\hline \multirow[t]{3}{*}{ Age in years } & $<20$ & $13(54.1)$ & II (45.8) & $3.34(1.31-8.49)$ & $1.25(0.23-6.75)$ & 0.313 \\
\hline & $20-34$ & $43(14.6)$ & $250(85.3)$ & $0.48(0.27-0.86)$ & $0.6 \mathrm{I}(0.27-\mathrm{I} .38)$ & 0.183 \\
\hline & $\geq 35$ & $23(26.1)$ & $65(73.8)$ & 1.0 & 1.0 & \\
\hline \multirow[t]{2}{*}{ Information about contraception } & Yes & $65(18.4)$ & $288(81.6)$ & 1.0 & 1.0 & \\
\hline & No & $14(26.9)$ & $38(73.1)$ & $1.63(0.84-3.19)$ & $1.24(0.25-6.09)$ & 0.730 \\
\hline \multirow[t]{2}{*}{ Residence } & Urban & $53(23.9)$ & $168(76.0)$ & $1.92(1.14-3.21)$ & $3.16(1.44-6.92)$ & 0.002 \\
\hline & Rural & $26(14.1)$ & $158(85.9)$ & 1.0 & 1.0 & \\
\hline \multirow[t]{2}{*}{ ANC in previous pregnancy } & No & $15(44.1)$ & $19(55.9)$ & $3.89(1.86-8.14)$ & $3.4(1.02-11.94)$ & 0.046 \\
\hline & Yes & $53(16.9)$ & $26 I(83 . I)$ & 1.0 & 1.0 & \\
\hline \multirow[t]{2}{*}{ Ever use contraceptive } & No & $49(23.2)$ & $162(76.8)$ & $1.64(0.99-2.72)$ & $1.77(0.80-3.95)$ & 0.196 \\
\hline & Yes & $30(15.5)$ & I $63(84.5)$ & 1.0 & 1.0 & \\
\hline \multirow{2}{*}{$\begin{array}{l}\text { Primary decision maker for family planning } \\
\text { use }\end{array}$} & Women herself & $38(12.2 \%)$ & $27 \mid(87.7 \%)$ & 1.0 & 1.0 & \\
\hline & Husband & $55(57.2 \%)$ & $4 \mathrm{I}(42.7 \%)$ & $3.10(1.86-5.19)$ & $2.85(1.18-6.88)$ & 0.020 \\
\hline \multirow{2}{*}{$\begin{array}{l}\text { Community education about maternal health } \\
\text { care }\end{array}$} & Yes & $25(11.5)$ & $193(88.5)$ & 1.0 & 1.0 & \\
\hline & No & 54 (28.9) & I33 (7I.I) & $3.13(1.86-5.29)$ & $2.36(1.06-5.27)$ & 0.040 \\
\hline \multirow{2}{*}{$\begin{array}{l}\text { Communicate with partner about family } \\
\text { planning }\end{array}$} & Yes & $49(20.4)$ & $191(79.6)$ & 1.0 & 1.0 & \\
\hline & No & $30(18.3)$ & I34 (8I.7) & $0.87(0.53-1.44)$ & $0.47(0.22-1.04)$ & 0.064 \\
\hline
\end{tabular}

Abbreviations: COR, crude odds ratio; AOR, adjusted odds ratio.

In addition, women who did not get community education about maternal health care had medium significance with an unintended pregnancy. A similar association between no exposure to community education and unintended pregnancy was also reported in a study at Gondar, Ethiopia. ${ }^{8}$ This finding indicates the importance of women's exposure to community education about maternal health services for women to have information about birth spacing, to understand the formal communication with health care providers on family planning services, thereby reducing unintended pregnancy.

Since this study was among pregnant women who attend ANC, there is a high tendency for the pregnancy to be intended. Thus, there might be an underestimation of unintended pregnancy. Therefore, the findings may not be generalizable to general populations. The cross-sectional nature prevents an effective causal analysis, and that the sample size may not be sufficient to generalize the results.

\section{Conclusion}

Nearly one-fifth of women had unintended pregnancy during the COVID 19 pandemic in public hospitals of southwest Ethiopia. Urban residence and not being the primary decision-maker had high significance with unintended pregnancy. Not having ANC in a previous pregnancy and not being exposed to community education about maternal health care had medium significance with unintended pregnancy. Strengthening women's decision-making power on family planning services and increasing women's access to contraceptives help to prevent unintended pregnancy. Efforts to address 
community education on maternal health services for all women are needed to reduce unintended pregnancy and its consequences in the future. Policy makers' strategy should focus on empowerment of women's autonomy on family planning usage and awareness creation of maternal health services.

\section{Data Sharing Statement}

The data set used or analyzed during this study is available from the corresponding author on reasonable request.

\section{Ethical Approval and Consent Participate}

This study was conducted per the Helsinki Declaration of research involving human subjects. ${ }^{31}$ In addition, the Research Ethical Review Board of the College of Medicine and Health Sciences, Wolkite University in Ethiopia approved this study. We submitted a support letter to participating hospitals and got permission. Data collection was anonymous to maintain the confidentiality of participants. Written informed consent was obtained from each study participant after explaining the purpose of the study.

\section{Acknowledgments}

We want to thank Worabe Comprehensive Specialized Hospital and Wolkite Specialized Teaching Hospital for giving us the necessary information, data collectors, and supervisors.

\section{Author Contributions}

All authors made substantial contributions to conception and design, acquisition of data, or analysis and interpretation of data; took part in drafting the article or revising it critically for important intellectual content; agreed to submit to the current journal; gave final approval for the version to be published; and agreed to be accountable for all aspects of the work.

\section{Funding}

There was no fund for this study.

\section{Disclosure}

The authors report no conflicts of interest in this work.

\section{References}

1. Yazdkhasti M, Pourreza A, Pirak A, et al. Unintended pregnancy and its adverse social and economic consequences on health system: a narrative review article. Iran J Public Health. 2015;44(1):12.

2. Kabir SM. Causes and consequences of unwanted pregnancy from Asian women's perspectives. Int J Gynaecol Obstet. 1989;30:9-14. doi:10.1016/ 0020-7292(89)90097-0

3. Bearak J, Popinchalk A, Ganatra B, et al. Unintended pregnancy and abortion by income, region, and the legal status of abortion: estimates from a comprehensive model for 1990-2019. Lancet Global Health. 2020;8(9):e1152-e1161. doi:10.1016/S2214-109X(20)30315-6

4. Kantorová V. Unintended pregnancy and abortion: what does it tell us about reproductive health and autonomy? Lancet Global Health. 2020;8(9): e1106-e1107. doi:10.1016/S2214-109X(20)30342-9

5. Claridge AM, Chaviano CL. Consideration of abortion in pregnancy: demographic characteristics, mental health, and protective factors. Women Health. 2013;53(8):777-794. doi:10.1080/03630242.2013.831018

6. Ameyaw EK, Budu E, Sambah F, et al. Prevalence and determinants of unintended pregnancy in sub-Saharan Africa: a multi-country analysis of demographic and health surveys. PLoS One. 2019;14(8):e0220970. doi:10.1371/journal.pone.0220970

7. Alene M, Yismaw L, Berelie Y, et al. Prevalence and determinants of unintended pregnancy in Ethiopia: a systematic review and meta-analysis of observational studies. PLoS One. 2020;15(4):e0231012. doi:10.1371/journal.pone.0231012

8. Asratie MH. Unintended pregnancy during COVID-19 pandemic among women attending antenatal care in Northwest Ethiopia: magnitude and associated factors. Int $J$ Womens Health. 2021;13:461. doi:10.2147/IJWH.S304540

9. Purdy C. Opinion: how will COVID-19 affect global access to contraceptives-and what can we do about it. Devex. 2020;10:2020.

10. Santoshini S. Family planning efforts upended by the coronavirus. Foreign Policy. 2020;13:2020.

11. Fund, U.N.P., J.H.U. Avenir Health, and V. University. Impact of the COVID-19 pandemic on family planning and ending gender-based violence, female genital mutilation and child marriage. New York: UNFPA; 2020.

12. Lindberg LD, VandeVusse A, Mueller J, et al. Early impacts of the COVID-19 pandemic: findings from the 2020 Guttmacher Survey of Reproductive Health Experiences. New York, NY: Guttmacher Institute; 2020. 
13. Riley T, Sully E, Ahmed Z, et al. Estimates of the potential impact of the COVID-19 pandemic on sexual and reproductive health in low-and middle-income countries. Int Perspect Sex Reprod Health. 2020;46:73-76. doi:10.1363/46e9020

14. Krubiner C, O'Donnell M, Kaufman J, et al. Addressing the COVID-19 Crisis's Indirect Health Impacts for Women and Girls. Center for Global Development; 2021:4.

15. Bahk J, Yun SC, Kim YM, Khang YH. Impact of unintended pregnancy on maternal mental health: a causal analysis using follow up data of the Panel Study on Korean Children (PSKC). BMC Pregnancy Childbirth. 2015;15(1):1-12. doi:10.1186/s12884-015-0429-z

16. Weldegebreal R, Melaku YA, Alemayehu M, et al. Unintended pregnancy among female sex workers in Mekelle city, northern Ethiopia: a cross-sectional study. BMC Public Health. 2015;15(1):1-9. doi:10.1186/1471-2458-15-1

17. Getu Melese K, Gebrie MH, Berta Badi M, et al. Unintended pregnancy in Ethiopia: community based cross-sectional study. Obstet Gynecol Int. 2016;2016. doi:10.1155/2016/4374791

18. Fite RO, Mohammedamin A, Abebe TW. Unintended pregnancy and associated factors among pregnant women in Arsi Negele Woreda, West Arsi Zone, Ethiopia. BMC Res Notes. 2018;11(1):1-7. doi:10.1186/s13104-018-3778-7

19. Teshome FT, Gebremariam A, Nigussie A. Prevalence of unintended pregnancy and associated factors among married pregnant women in Ganji woreda, west Wollega Oromia region, Ethiopia. Sci J Public Health. 2014;2:92-101.

20. Geda NR, Lako TK. A population based study on unintended pregnancy among married women in a district in Southern Ethiopia. $J$ Geogr Reg Plann. 2011;4(7):417-427.

21. Tsegaye AT, Mengistu M, Shimeka A. Prevalence of unintended pregnancy and associated factors among married women in west Belessa Woreda, Northwest Ethiopia, 2016. Reprod Health. 2018;15(1):1-8. doi:10.1186/s12978-018-0649-6

22. Kassahun EA, Zeleke LB, Dessie AA, et al. Factors associated with unintended pregnancy among women attending antenatal care in Maichew Town, Northern Ethiopia, 2017. BMC Res Notes. 2019;12(1):1-6. doi:10.1186/s13104-019-4419-5

23. Santelli J, Rochat R, Hatfield-Timajchy K, et al. The measurement and meaning of unintended pregnancy. Perspect Sex Reprod Health. 2003;35 (2):94-101. doi:10.1363/3509403

24. Getachew FD. Level of unintended pregnancy and its associated factors among currently pregnant women in Duguna Fango district, Wolaita zone, southern Ethiopia. Malays J Med Biol Res. 2016;3(1):11-24. doi:10.18034/mjmbr.v3i1.400

25. Lamina MA. Prevalence and determinants of unintended pregnancy among women in South-Western Nigeria. Ghana Med J. 2015;49(3):187-194. doi:10.4314/gmj.v49i3.10

26. Nyarko SH. Unintended pregnancy among pregnant women in Ghana: prevalence and predictors. J Pregnancy. 2019;2019:1-8. doi:10.1155/2019/ 2920491

27. Joyce T, Kaestner R, Korenman S. The stability of pregnancy intentions and pregnancy-related maternal behaviors. Matern Child Health J. 2000;4 (3):171-178. doi:10.1023/A:1009571313297

28. Sarder A, Islam SMS, Talukder A, Ahammed B. Prevalence of unintended pregnancy and its associated factors: evidence from six south Asian countries. PLoS One. 2021;16(2):e0245923. doi:10.1371/journal.pone.0245923

29. Gite A, Liulseged N, Seyife H, et al. Unintended pregnancy: magnitude and associated factors among pregnant women in Arba Minch Town, Gamo Gofa Zone, Ethiopia, 2015. Reprod Syst Sex Disord. 2016;5(04):1-6. doi:10.4172/2161-038X.1000193

30. Gizaw W, Fetene G, Mohammed S, et al. Magnitude of unplanned pregnancy and its associated factors among pregnant women attending antenatal care at Tepi General Hospital Sheka Zone, Southwest Ethiopia, 2017. Insights Reprod Med. 2018;2(2):1.

31. World Medical Association. World Medical Association Declaration of Helsinki. Ethical principles for medical research involving human subjects. Bull World Health Organ. 2001;79(4):373.

\section{Publish your work in this journal}

Open Access Journal of Contraception is an international, peer-reviewed, open access, online journal, publishing original research, reports, reviews and commentaries on all areas of contraception. In addition to clinical research, demographics and health-related aspects, the journal welcomes new findings in animal and preclinical studies relating to understanding the biological mechanisms and practical development of new contraceptive agents. The manuscript management system is completely online and includes a very quick and fair peer-review system. Visit http://www.dovepress.com/testimonials.php to read real quotes from published authors.

Submit your manuscript here: http://www.dovepress.com/open-access-journal-of-contraception-journal 